\title{
MISALIGNMENT OF MAGNETIC FIELDS AND OUTFLOWS IN PROTOSTELLAR CORES
}

\author{
Charles L. H. Hull ${ }^{1}$, Richard L. Plambeck ${ }^{1}$, Alberto D. Bolatto ${ }^{2}$, Geoffrey C. Bower ${ }^{1}$, John M. Carpenter ${ }^{3}$, \\ Richard M. Crutcher ${ }^{4}$, Jason D. Fiege ${ }^{5}$, Erica Franzmann ${ }^{5}$, Nicholas S. Hakobian ${ }^{4}$, Carl Heiles ${ }^{1}$, Martin Houde Hed, $^{6,7}$, \\ A. Meredith Hughes ${ }^{1,8,15}$, Katherine Jameson ${ }^{2}$, Woojin Kwon ${ }^{4,9}$, James W. Lamb ${ }^{3}$, Leslie W. Looney ${ }^{4,10}$, \\ Brenda C. Matthews ${ }^{11,12}$, Lee Mundy ${ }^{2}$, Thushara Pillai ${ }^{3}$, Marc W. Pound ${ }^{2}$, Ian W. Stephens ${ }^{4}$, John J. Tobin ${ }^{10,16}$, \\ John E. Vaillancourt ${ }^{13}$, N. H. Volgenau ${ }^{14}$, and Melvyn C. H. Wright ${ }^{1}$ \\ ${ }^{1}$ Astronomy Department \& Radio Astronomy Laboratory, University of California, Berkeley, CA 94720-3411, USA; chat@astro.berkeley.edu \\ ${ }^{2}$ Astronomy Department \& Laboratory for Millimeter-wave Astronomy, University of Maryland, College Park, MD 20742, USA \\ ${ }^{3}$ Department of Astronomy, California Institute of Technology, 1200 E. California Blvd., MC 249-17, Pasadena, CA 91125, USA \\ ${ }^{4}$ Department of Astronomy, University of Illinois at Urbana-Champaign, 1002 W Green Street, Urbana, IL 61801, USA \\ 5 Department of Physics \& Astronomy, University of Manitoba, Winnipeg, MB R3T 2N2, Canada \\ ${ }^{6}$ Department of Physics \& Astronomy, University of Western Ontario, London, ON N6A 3K7, Canada \\ ${ }^{7}$ Division of Physics, Mathematics, \& Astronomy, California Institute of Technology, Pasadena, CA 91125, USA \\ 8 Van Vleck Observatory, Astronomy Department, Wesleyan University, 96 Foss Hill Drive, Middletown, CT 06459, USA \\ ${ }^{9}$ SRON Netherlands Institute for Space Research, Landleven 12, 9747 AD Groningen, The Netherlands \\ ${ }^{10}$ National Radio Astronomy Observatory, 520 Edgemont Rd., Charlottesville, VA 22903, USA \\ ${ }^{11}$ Department of Physics \& Astronomy, University of Victoria, 3800 Finnerty Rd., Victoria, BC V8P 5C2, Canada \\ ${ }^{12}$ National Research Council of Canada, 5071 West Saanich Rd., Victoria, BC V9E 2E7, Canada \\ 13 SOFIA Science Center, Universities Space Research Association, NASA Ames Research Center, Moffett Field, CA 94035, USA \\ ${ }^{14}$ Combined Array for Research in Millimeter-wave Astronomy, Owens Valley Radio Observatory, P.O. Box 968, Big Pine, CA 93513, USA \\ Received 2013 February 22; accepted 2013 March 28; published 2013 April 25
}

\begin{abstract}
We present results of $\lambda 1.3 \mathrm{~mm}$ dust-polarization observations toward 16 nearby, low-mass protostars, mapped with $\sim 2$. 5 resolution at CARMA. The results show that magnetic fields in protostellar cores on scales of $\sim 1000$ AU are not tightly aligned with outflows from the protostars. Rather, the data are consistent with scenarios where outflows and magnetic fields are preferentially misaligned (perpendicular), or where they are randomly aligned. If one assumes that outflows emerge along the rotation axes of circumstellar disks, and that the outflows have not disrupted the fields in the surrounding material, then our results imply that the disks are not aligned with the fields in the cores from which they formed.
\end{abstract}

Key words: ISM: magnetic fields - magnetic fields - polarization - stars: formation - stars: magnetic field - stars: protostars

Online-only material: color figure

\section{INTRODUCTION}

Optical polarization measurements of background stars show that magnetic fields are well ordered on large scales in the low-density envelopes of molecular clouds, as in the Pipe Nebula (Franco et al. 2010), which suggests that these parsec-scale envelopes are magnetically supported ("subcritical"). Ultimately, however, ambipolar diffusion (e.g., Fiedler \& Mouschovias 1993), turbulence (e.g., Mac Low \& Klessen 2004), or turbulent magnetic reconnection diffusion (Lazarian 2005; Leão et al. 2012) allow the formation of "supercritical" dense cores in which gravity overwhelms magnetic support. In the simplest axisymmetric case, one expects the field lines to be drawn into an hourglass shape by gravitational collapse, forming a 1000 AU diameter "pseudodisk" (Galli \& Shu 1993).

Although the magnetic field ( $B$-field) may not be strong enough to prevent the formation of a protostar within the pseudodisk, it can have a significant impact on the accretion rate onto the star, and on the formation of a rotationally supported circumstellar disk in which planets will form. In the limiting case of flux freezing, the field close to the protostar becomes strong enough to brake the rotation of the infalling gas completely, preventing the formation of a rotationally supported disk (Galli

\footnotetext{
15 Miller Fellow.

${ }^{16}$ Hubble Fellow.
}

et al. 2006). Even if ambipolar diffusion allows the field to escape the central core, the redistributed flux tends to be trapped in a ring surrounding the star, greatly reducing the infall rate $(\mathrm{Li}$ et al. 2011).

Magnetic braking is less effective and disks should form more easily if the rotation axis of the cloud is at an angle to the magnetic field (Joos et al. 2012; Krumholz et al. 2013). Indeed, from an observational perspective it is clear that circumstellar disks typically are not well aligned with the parsec-scale magnetic fields in the surrounding molecular cloud. If they were, the bipolar outflows and jets that emerge along the axes of these disks would all be parallel with the ambient $B$-field, but this is not observed: Ménard \& Duchêne (2004) have shown that the optical jets from classical T Tauri stars in the Taurus-Auriga molecular cloud are randomly oriented with respect to the parsec-scale magnetic field in this cloud; Targon et al. (2011) obtained a similar result for 28 regions spread over the Galaxy, although they do find some evidence for the alignment of jets from younger, Class 0 and Class I, protostars.

Polarization observations of background stars are unable to probe the magnetic field morphologies inside the dense cores where circumstellar disks form; even at infrared wavelengths the extinction through these regions is too great. Mapping the polarized thermal emission from dust grains at millimeter and submillimeter wavelengths is the usual means of studying the magnetic fields in these regions. Under most circumstances, 
spinning dust grains are expected to align themselves with their long axes perpendicular to the magnetic field (Hoang \& Lazarian 2009), so normally the thermal radiation from these grains is weakly polarized perpendicular to the field.

Dust-polarization maps of many sources have been obtained at submillimeter wavelengths with single-dish instruments, e.g., with the SCUBA polarimeter on the James Clerk Maxwell telescope at $850 \mu \mathrm{m}$ (Matthews et al. 2009), and with the Hertz polarimeter on the Caltech Submillimeter Observatory (CSO) at $350 \mu \mathrm{m}$ (Dotson et al. 2010). These maps have angular resolutions of about 20", corresponding to scales of 3000-8000 AU in nearby molecular clouds. Curran \& Chrysostomou (2007) found that on these scales outflows and inferred $B$-fields are randomly aligned.

Higher angular resolution is required to study the field geometry in the innermost regions of the cores where circumstellar disks form. Thus far, interferometric polarization maps with resolutions of a few arcseconds have been published for about a dozen sources, using data from the Owens Valley Radio Observatory, the Berkeley-Illinois-Maryland Array, and the Submillimeter Array (SMA). Most of this work has focused on detailed analyses of individual objects: examples include maps of NGC 1333-IRAS 4A (Girart et al. 2006), IRAS 16293 (Rao et al. 2009), and Orion KL (Rao et al. 1998; Tang et al. 2010). In this limited set of sources, outflows were often found to be skewed with respect to the inferred magnetic field directions, hinting that circumstellar disks may not be tightly aligned with the magnetic fields on $\sim 1000$ AU scales.

The $1 \mathrm{~mm}$ dual-polarization receiver system at CARMA (the Combined Array for Research in Millimeter-wave Astronomy) allows us to map the dust polarization toward many more sources. Here we present results from the TADPOL ${ }^{17}$ survey, a CARMA key project to study dust polarization in protostellar cores. This paper focuses on results from nearby, lowmass protostars. We compare the field direction inferred from dust-polarization measurements with the outflow direction, which indicates the axis of the rotationally supported disk. On the $\sim 1000$ AU scales probed by our data, magnetic fields appear to be either preferentially misaligned (perpendicular) or randomly aligned with respect to outflows.

\section{OBSERVATIONS AND DATA REDUCTION}

Observations were made with CARMA between 2011 May and 2012 October. We selected sources from catalogs of young stellar objects, including Jørgensen et al. (2007), Matthews et al. (2009), Tobin et al. (2010), and Enoch et al. (2011). We focus on Class 0 and Class I objects at distances of $d \lesssim 400 \mathrm{pc}$ that are known to have bipolar outflows.

The polarization system consists of dual-polarization receivers that are sensitive to right- (R) and left-circular (L) polarization, and a spectral-line correlator that measures all four cross polarizations (RR, LL, LR, and RL) on the 105 baselines connecting the 15 antennas. The receivers comprise a single feed horn, a waveguide circular polarizer (Plambeck \& Engargiola 2010), an orthomode transducer (Navarrini \& Plambeck 2006), and two mixers. The receivers are doublesideband; a phase-switching pattern applied to the local oscillator (LO) allows signals in the lower (LSB) and upper sidebands (USB) to be separated in the correlator.

For these observations, the correlator was set up with three $500 \mathrm{MHz}$ wide bands to measure the dust continuum and one

\footnotetext{
17 Telescope Array Doing POLarization: http://tadpol.astro.illinois.edu/
}

$31 \mathrm{MHz}$ wide band to map bipolar outflows. The frequency of the first LO was $223.821 \mathrm{GHz}$. The three continuum bands were centered at $6.0,7.5$, and $8.0 \mathrm{GHz}$ in the intermediate frequency (IF). The corresponding RF frequencies are equal to the difference (LSB) or the sum (USB) of the LO and the IF. The spectral-line band was centered at IF $=6.717 \mathrm{GHz}$, allowing simultaneous observations of the $\mathrm{SiO}(5-4)$ line in the LSB and the $\mathrm{CO}(2-1)$ line in the USB. For the spectral-line measurements, the channel spacing is $0.2 \mathrm{~km} \mathrm{~s}^{-1}$.

In addition to the usual gain, passband, and flux calibrations, polarization observations require two additional calibrations: "XYphase" and leakage. The XYphase calibration corrects for phase differences between the $\mathrm{L}$ and $\mathrm{R}$ receivers, and is done by observing an artificially polarized noise source of known position angle. The leakage corrections compensate for crosscoupling between the $\mathrm{L}$ and $\mathrm{R}$ receivers, and are calibrated by observing a strong source (usually the gain calibrator) over a range of parallactic angles. There are no moving parts in the CARMA dual-polarization receivers, so the measured leakages are stable with time. A typical antenna has a band-averaged leakage amplitude (i.e., a voltage coupling from L into R, or vice versa) of $6 \%$. We perform calibration and imaging with the MIRIAD data reduction package (Sault et al. 1995). Using multi-frequency synthesis and natural weighting, we create dust-continuum maps of all four Stokes parameters $(I, Q, U, V)$. The typical beam size is 2 . 5 , which corresponds to a resolution of $750 \mathrm{AU}$ at a distance of $300 \mathrm{pc}$. We produce polarization position-angle and intensity maps from the Stokes $I, Q$, and $U$ data, where the position angle of the incoming radiation is $\chi=0.5 \arctan (U / Q)$, and the bias-corrected polarized intensity is $P_{\mathrm{c}}=\sqrt{Q^{2}+U^{2}-\sigma_{\mathrm{P}}^{2}}\left(\sigma_{\mathrm{P}}\right.$ is the median rms noise in the Stokes $Q$ and $U$ maps; Vaillancourt 2006).

In good weather $\sigma_{\mathrm{P}} \approx 0.4 \mathrm{mJy}$ beam $^{-1}$ for a single $6 \mathrm{hr}$ observation, and can be as low as $\sim 0.2 \mathrm{mJy}^{\text {beam }^{-1}}$ when multiple observations are combined. We consider it a detection only if $P_{\mathrm{c}} \geqslant 3 \sigma_{\mathrm{P}}$ and the location of the polarized emission coincides with a detection of total intensity $I \geqslant 2 \sigma_{\mathrm{I}}$, where $\sigma_{\mathrm{I}}$ is the rms noise in the Stokes I map.

We also generate maps of the red- and blueshifted $\mathrm{CO}$ and $\mathrm{SiO}$ line wings to measure outflow directions. We generally use CO maps to measure the outflow direction, but occasionally we use $\mathrm{SiO}$ if the $\mathrm{CO}$ emission is too complex. We do not attempt to measure polarization in the spectral-line data because of fine-scale frequency structure in the polarization leakages.

\section{RESULTS AND ANALYSIS}

Of the 27 TADPOL sources within $\sim 400 \mathrm{pc}$, we detected dust polarization toward 16, which we focus on in this paper. The full set of TADPOL results will be presented in a separate paper.

Figure 1 shows some example results. In all of the plots, the dust-polarization vectors have been rotated by $90^{\circ}$ to show the inferred magnetic field direction and are plotted at the Nyquist spatial frequency (two vectors per synthesized beam).

Table 1 lists the results for the 16 TADPOL sources, as well as for IRAS 16293, which was previously published by Rao et al. (2009). Two of the sources each have two distinct outflows, which we consider as independent data, thus making a total of 19 entries. Note that NGC 1333-IRAS 4A, one of the sources in the TADPOL sample, has been mapped in detail previously (Girart et al. 2006) and was included in our survey as a cross-check. The polarization directions at the intensity peaks of the $230 \mathrm{GHz}$ CARMA map and the previously published $345 \mathrm{GHz}$ SMA map are in excellent agreement. 

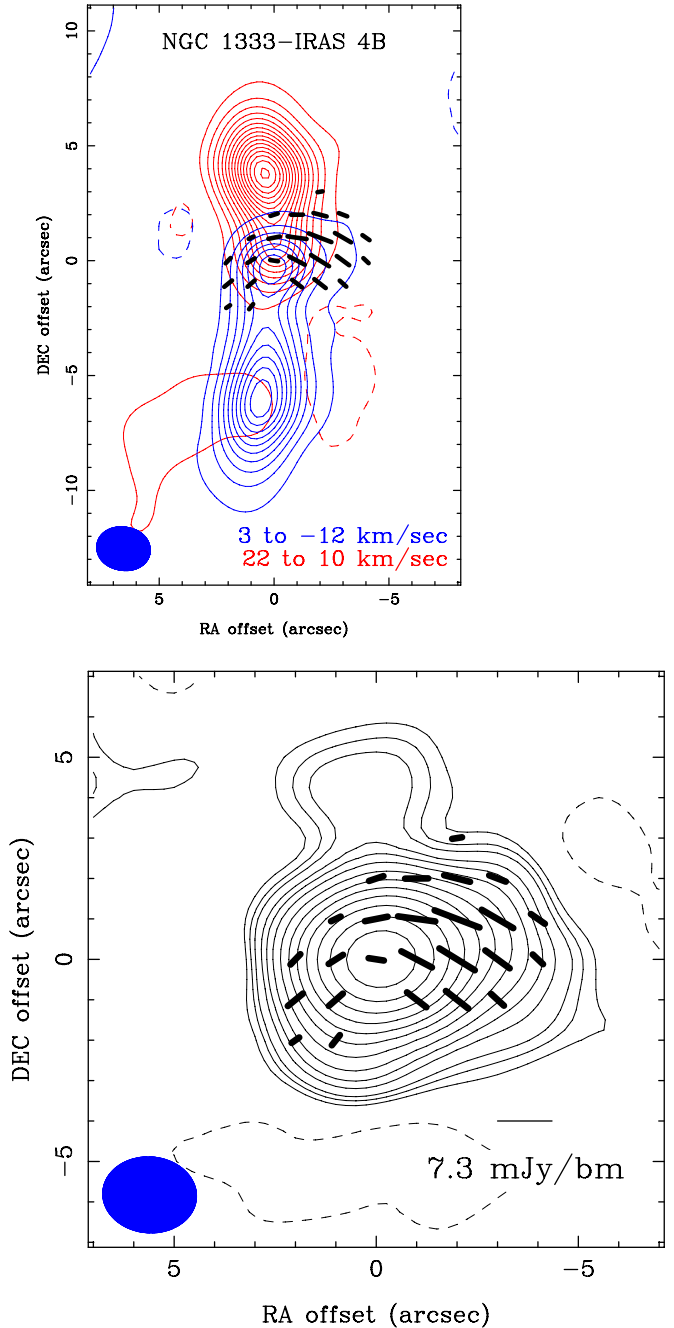
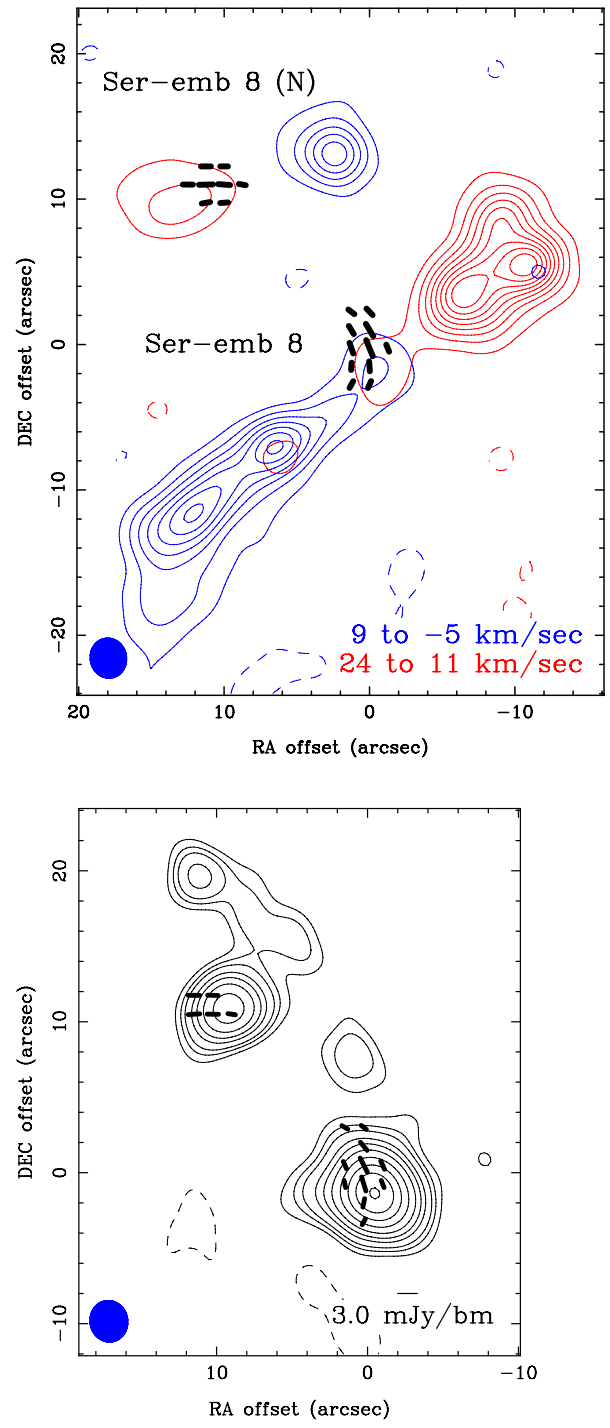

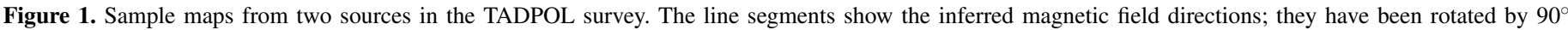

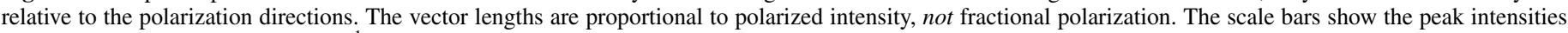

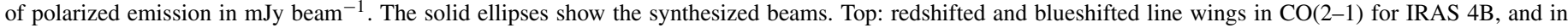

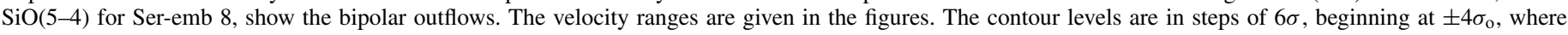

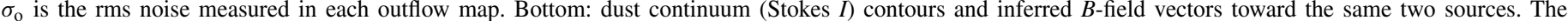

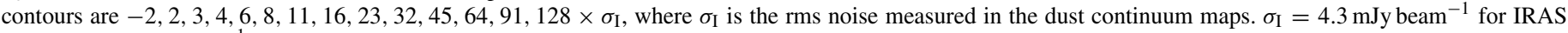
$4 \mathrm{~B} ; \sigma_{\mathrm{I}}=3.3 \mathrm{mJy}$ beam $^{-1}$ for Ser-emb 8 .

(A color version of this figure is available in the online journal.)

To estimate the outflow direction, we measure the position angles of lines connecting the center of the continuum source and the intensity peaks of the red and blue outflow lobes; we take the average of the two position angles as the outflow direction. As a crude estimate of the uncertainty $\sigma_{\mathrm{o}}$, we use half the difference of the position angles. The $B$-field direction is calculated by averaging the $B$-field position angles, weighted by the Stokes $I$ total intensity, which gives the field direction in the densest part of the core. The uncertainty in the $B$-field $\sigma_{\mathrm{B}}$ is the average of the $B$-field position angle uncertainties, also weighted by the Stokes $I$ total intensity. The total uncertainty in the angle between the $B$-field and the outflow is $\sigma_{\mathrm{o}-\mathrm{B}}=\sqrt{\sigma_{\mathrm{o}}^{2}+\sigma_{\mathrm{B}}^{2}}$.

Figure 2 shows the cumulative distribution function (CDF) of the projected angles between the $B$-fields and outflows of the sources in Table 1 . The $B$-field and outflow position angles we observe are projected onto the plane of the sky. To determine if the large scatter in position angle differences could be due to projection effects, we compare the results with Monte Carlo simulations where the outflows and $B$-fields are tightly aligned, preferentially misaligned (perpendicular), or randomly aligned.

For the tightly aligned case, the simulation randomly selects pairs of vectors in three dimensions that are within $20^{\circ}$ of one another, and then projects the vectors onto the plane of the sky and measures their angular differences. The resulting CDF is shown by the upper dotted curve in Figure 2. In this case projection effects are not as problematic as one might think: to have a projected separation larger than $20^{\circ}$, the two vectors must point almost along the line of sight.

For the preferentially misaligned case, the simulation randomly selects pairs of vectors that are separated by $70^{\circ}-90^{\circ}$. The resulting CDF is shown by the lower dotted curve in Figure 2. In this case projection effects are more important, 
Table 1

Observations

\begin{tabular}{|c|c|c|c|c|c|c|c|c|}
\hline Source & $\begin{array}{c}\alpha \\
(\mathrm{J} 2000)\end{array}$ & $\begin{array}{c}\delta \\
(\mathrm{J} 2000)\end{array}$ & $\begin{array}{c}\chi_{\mathrm{B}}\left(\sigma_{\mathrm{B}}\right) \\
\left(^{\circ}\right)\end{array}$ & $\begin{array}{c}\chi_{\mathrm{o}}\left(\sigma_{\mathrm{o}}\right) \\
\left({ }^{\circ}\right)\end{array}$ & $\begin{array}{c}\theta_{\mathrm{O}-\mathrm{B}}\left(\sigma_{\mathrm{O}-\mathrm{B}}\right) \\
\left({ }^{\circ}\right)\end{array}$ & $\begin{array}{c}\theta_{\mathrm{bm}} \\
\left({ }^{\prime \prime}\right)\end{array}$ & $\begin{array}{c}d \\
(\mathrm{pc})\end{array}$ & $\begin{array}{l}\text { Distance } \\
\text { Ref. }^{\text {d }}\end{array}$ \\
\hline L1448 IRS 2 & $03: 25: 22.4$ & $30: 45: 13.2$ & 139(9) & 134(5) & $5(10)$ & 3.63 & 230 & 1 \\
\hline L1448N(B) & $03: 25: 36.3$ & $30: 45: 14.8$ & $31(6)$ & $97(2)$ & $66(6)$ & 2.04 & 230 & 1 \\
\hline NGC 1333 -IRAS $2 \mathrm{~A}^{\mathrm{a}}$ & 03:28:55.6 & $31: 14: 37.1$ & $\begin{array}{l}82(6) \\
82(6)\end{array}$ & $\begin{array}{l}98(6) \\
21(9)\end{array}$ & $\begin{array}{c}16(8) \\
61(11)\end{array}$ & 3.45 & 230 & $\begin{array}{l}1 \\
1\end{array}$ \\
\hline NGC 1333-IRAS 4A & $03: 29: 10.5$ & $31: 13: 31.3$ & $58(2)$ & $18(10)$ & $40(10)$ & 2.52 & 230 & 1 \\
\hline NGC 1333-IRAS 4B & $03: 29: 12.0$ & 31:13:08.1 & $86(5)$ & $0(5)$ & $86(7)$ & 2.09 & 230 & 1 \\
\hline HH 211 & $03: 43: 56.8$ & $32: 00: 50.0$ & $164(6)$ & $116(1)$ & $48(6)$ & 3.95 & 230 & 1 \\
\hline L1551 IRS 5 & $04: 31: 44.5$ & $18: 08: 31.5$ & $165(4)$ & $67(5)$ & $82(6)$ & 2.18 & 140 & 2 \\
\hline L1527 & 04:39:53.9 & 26:03:09.6 & $174(8)$ & $92(7)$ & $82(11)$ & 3.06 & 140 & 2 \\
\hline OMC3-MMS5 $5^{\mathrm{b}}$ & $05: 35: 22.4$ & $-05: 01: 14.5$ & $52(9)$ & $80(6)$ & $28(11)$ & 3.22 & 415 & 3 \\
\hline OMC3-MMS6 & $05: 35: 23.4$ & $-05: 01: 30.6$ & $45(2)$ & $171(8)$ & $54(8)$ & 3.22 & 415 & 3 \\
\hline VLA 1623 & $16: 26: 26.4$ & $-24: 24: 30.4$ & $36(9)$ & $120(5)$ & $84(10)$ & 2.89 & 120 & 4 \\
\hline \multirow[t]{2}{*}{ IRAS $16293 \mathrm{~A}^{\mathrm{a}, \mathrm{c}}$} & $16: 32: 22.9$ & $-24: 28: 36.3$ & $5(9)$ & $77(9)$ & $72(13)$ & 2.46 & 178 & 5 \\
\hline & & & $5(9)$ & 143(9) & $42(13)$ & & & 5 \\
\hline Ser-emb 8 & $18: 29: 48.1$ & 01:16:43.6 & $39(6)$ & $107(1)$ & $68(6)$ & 2.63 & 415 & 6 \\
\hline Ser-emb $8(N)$ & $18: 29: 48.7$ & 01:16:55.8 & $88(7)$ & $129(2)$ & $41(7)$ & 2.63 & 415 & 6 \\
\hline Ser-emb 6 & $18: 29: 49.8$ & $01: 15: 20.3$ & $157(3)$ & $135(3)$ & $22(4)$ & 2.71 & 415 & 6 \\
\hline L1157 & 20:39:06.2 & 68:02:16.0 & $146(4)$ & $146(7)$ & $0(8)$ & 2.39 & 250 & 7 \\
\hline CB 230 & $21: 17: 38.7$ & $68: 17: 32.4$ & $89(6)$ & $172(4)$ & $83(7)$ & 3.05 & 400 & 8 \\
\hline
\end{tabular}

Notes. Coordinates are fitted positions of dust emission peaks. The outflow angle $\chi_{\mathrm{o}}$ and inferred magnetic-field angle $\chi_{\mathrm{B}}$ are measured counterclockwise from north. The angle difference $\theta_{\mathrm{O}-\mathrm{B}}$ between the outflow and the $B$-field is always between $0^{\circ}$ and $90^{\circ}$. The $B$-field direction is assumed to be perpendicular to the direction of the polarized dust emission. $d$ is the distance to the source. $\theta_{\mathrm{bm}}$ is the geometric mean of the major and minor axes of the synthesized beam.

${ }^{\text {a }}$ Source has two outflows.

${ }^{\mathrm{b}}$ Coordinates from Takahashi et al. (2009).

${ }^{\mathrm{c}}$ Results from Rao et al. (2009).

d Distance references. (1) Hirota et al. 2011; (2) Loinard et al. 2007; (3) Menten et al. 2007; (4) Loinard et al. 2008; (5) Imai et al. 2007; (6) Dzib et al. 2010; (7) Looney et al. 2007; (8) Launhardt et al. 2010.

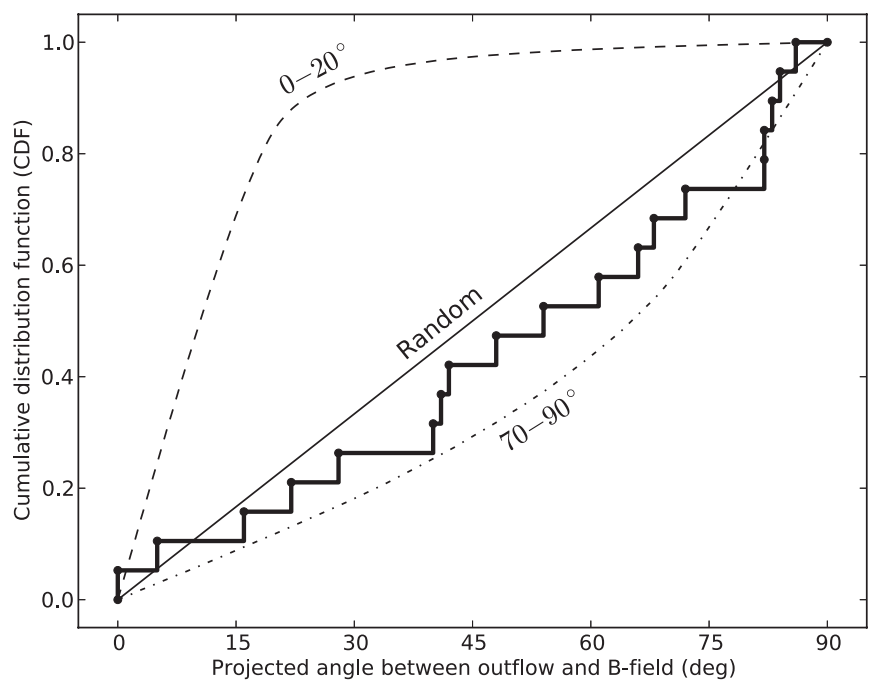

Figure 2. Thick solid curve shows the cumulative distribution function (CDF) of the (projected) angles between the mean magnetic field and outflow directions for the sources in Table 1. The upper dashed curve is the CDF from a Monte Carlo simulation where outflow and $B$-field directions are oriented within $20^{\circ}$ of one another (tightly aligned). The lower dot-dashed curve is the CDF from a simulation where outflow and $B$-field directions are separated by $70^{\circ}-90^{\circ}$ (preferentially misaligned). The straight line is the $\mathrm{CDF}$ for random orientation.

and result in a CDF that is similar to that expected for random alignment, shown by the solid curve.

A Kolmogorov-Smirnov (K-S) test shows that the probability that our data were drawn from the same population as the tightly aligned model is $3 \times 10^{-9}$, ruling out this scenario. The probability that the results were drawn from a preferentially misaligned population is 0.79 , and from a random population is 0.64 . Although the probability is slightly higher for the misaligned case, either of the latter two models are consistent with the data.

\section{DISCUSSION}

Most analytical models of star formation assume that the rotation axis of a protostellar core, its magnetic field direction, and its outflow direction all are parallel (Shu et al. 2000; Konigl \& Pudritz 2000). Our results appear to contradict this simple picture.

We have assumed that dust grains always are aligned with their long axes perpendicular to the magnetic fields, and thus that $B$-fields are perpendicular to the polarization directions. This may not always be the case. For example, if grains are mechanically aligned by outflows, then the polarization is expected to be parallel to the $B$-field (Gold 1952). Mechanical alignment should affect only a small portion of a protostellar core, however, as the maximum opening angle of the outflows in our sample is $\sim 60^{\circ}$, corresponding to $\sim 10 \%$ of the core volume. Lazarian (2007) and Hoang \& Lazarian (2009) show that grains aligned by radiative torques can also be stably aligned parallel to the $B$-field under some conditions, but this is unlikely in the densest part of a protostellar core.

Outflows may also affect magnetic field morphologies. Because of magnetic tension, the influence of an outflow is not restricted to the outflow cavity and potentially could extend over a significant fraction of the core volume. Additionally, simulations by Tomisaka (2011) have shown that $B$-field morphologies 
can depend on the outflow launching mechanism: the $B$-field is predominantly toroidal in a magnetocentrifugally driven wind, and is predominantly poloidal in a jet-driven outflow with entrained molecular material. These differences in morphology, combined with projection effects, could result in random orientations between outflows and $B$-fields.

Finally, some simulations, such as those by Hennebelle \& Ciardi (2009), Joos et al. (2012), and Li et al. (2013) suggest that misalignment of the $B$-field and the core rotation axis can aid in the formation of circumstellar disks, given a mass-toflux ratio in the core of $\gtrsim 2$. Li et al. (2011) find that disk formation is suppressed in the case where fields and core rotation axes are parallel, even when non-ideal MHD effects are considered. Hence, these models suggest that misalignment may be a necessary condition for the formation of disks.

\section{SUMMARY}

The results from the TADPOL survey show that magnetic fields on scales of $\sim 1000$ AU are not tightly aligned with protostellar outflows. Rather, the data are consistent both with scenarios where outflows and magnetic fields are preferentially misaligned (perpendicular), and where they are randomly aligned. If one assumes that outflows emerge along the rotation axes of circumstellar disks, and that the outflows have not disrupted the fields in the surrounding material, then our results imply that the disks are not aligned with the fields in the cores from which they formed.

It could be fruitful to investigate whether alignment correlates with core rotation, field strength, outflow velocity, multiplicity, or age. Higher resolution polarization observations with ALMA will test these correlations at the $\sim 100$ AU scale of circumstellar disks.

We thank the referee for insightful comments.

C.L.H.H. thanks Chris McKee, Richard Klein, Mark Krumholz, and Andrew Myers for a helpful discussion, and acknowledges the advice and guidance of the members of the Berkeley Radio Astronomy Laboratory and the Berkeley Astronomy Department.

C.L.H.H. acknowledges support from an NSF Graduate Fellowship. J.D.F. acknowledges support from an NSERC Discovery grant. J.J.T. acknowledges support provided by NASA through Hubble Fellowship grant \#HST-HF-51300.01-A awarded by the Space Telescope Science Institute, which is operated by the Association of Universities for Research in Astronomy, Inc., for NASA, under contract NAS 5-26555. A.D.B. acknowledges support from a CAREER grant NSF-AST0955836, NSF-AST1139998, and a Research Corporation for Science Advancement Cottrell Scholar award.

Support for CARMA construction was derived from the states of California, Illinois, and Maryland, the James S. McDonnell Foundation, the Gordon and Betty Moore Foundation, the Kenneth T. and Eileen L. Norris Foundation, the University of Chicago, the Associates of the California Institute of
Technology, and the National Science Foundation. Ongoing CARMA development and operations are supported by the National Science Foundation under a cooperative agreement, and by the CARMA partner universities.

\section{REFERENCES}

Curran, R. L., \& Chrysostomou, A. 2007, MNRAS, 382, 699

Dotson, J. L., Vaillancourt, J. E., Kirby, L., et al. 2010, ApJS, 186, 406

Dzib, S., Loinard, L., Mioduszewski, A. J., et al. 2010, ApJ, 718, 610

Enoch, M. L., Corder, S., Duchêne, G., et al. 2011, ApJS, 195, 21

Fiedler, R. A., \& Mouschovias, T. C. 1993, ApJ, 415, 680

Franco, G. A. P., Alves, F. O., \& Girart, J. M. 2010, ApJ, 723, 146

Galli, D., Lizano, S., Shu, F. H., \& Allen, A. 2006, ApJ, 647, 374

Galli, D., \& Shu, F. H. 1993, ApJ, 417, 243

Girart, J. M., Rao, R., \& Marrone, D. P. 2006, Sci., 313, 812

Gold, T. 1952, MNRAS, 112, 215

Hennebelle, P., \& Ciardi, A. 2009, A\&A, 506, L29

Hirota, T., Honma, M., Imai, H., et al. 2011, PASJ, 63, 1

Hoang, T., \& Lazarian, A. 2009, ApJ, 697, 1316

Imai, H., Nakashima, K., Bushimata, T., et al. 2007, PASJ, 59, 1107

Joos, M., Hennebelle, P., \& Ciardi, A. 2012, A\&A, 543, A128

Jørgensen, J. K., Bourke, T. L., Myers, P. C., et al. 2007, ApJ, 659, 479

Konigl, A., \& Pudritz, R. E. 2000, in Protostars and Planets IV, ed. V. Mannings, A. P. Boss, \& S. S. Russell (Tucson, AZ: Univ. Arizona Press), 759

Krumholz, M. R., Crutcher, R. M., \& Hull, C. L. H. 2013, ApJL, 767, L11

Launhardt, R., Nutter, D., Ward-Thompson, D., et al. 2010, ApJS, 188, 139

Lazarian, A. 2005, in AIP Conf. Ser. 784, Magnetic Fields in the Universe: From Laboratory and Stars to Primordial Structures., ed. E. M. de Gouveia dal Pino, G. Lugones, \& A. Lazarian (Melville, NY: AIP), 42

Lazarian, A. 2007, JQSRT, 106, 225

Leão, M. R. M., de Gouveia Dal Pino, E. M., Santos-Lima, R., \& Lazarian, A. 2012, arXiv:1209.1846

Li, Z.-Y., Krasnopolsky, R., \& Shang, H. 2011, ApJ, 738, 180

Li, Z.-Y., Krasnopolsky, R., \& Shang, H. 2013, arXiv:1301.6545

Loinard, L., Torres, R. M., Mioduszewski, A. J., et al. 2007, ApJ, 671, 546

Loinard, L., Torres, R. M., Mioduszewski, A. J., \& Rodríguez, L. F. 2008, ApJL, 675, L29

Looney, L. W., Tobin, J. J., \& Kwon, W. 2007, ApJL, 670, L131

Mac Low, M.-M., \& Klessen, R. S. 2004, RvMP, 76, 125

Matthews, B. C., McPhee, C. A., Fissel, L. M., \& Curran, R. L. 2009, ApJS, 182,143

Ménard, F., \& Duchêne, G. 2004, A\&A, 425, 973

Menten, K. M., Reid, M. J., Forbrich, J., \& Brunthaler, A. 2007, A\&A, 474,515

Navarrini, A., \& Plambeck, R. 2006, ITMTT, 54, 272

Plambeck, R., \& Engargiola, G. 2010, CARMA Memos, 54

Rao, R., Crutcher, R. M., Plambeck, R. L., \& Wright, M. C. H. 1998, ApJL, 502, L75

Rao, R., Girart, J. M., Marrone, D. P., Lai, S.-P., \& Schnee, S. 2009, ApJ, 707, 921

Sault, R. J., Teuben, P. J., \& Wright, M. C. H. 1995, in ASP Conf. Ser. 77, Astronomical Data Analysis Software and Systems IV (San Francisco, CA: ASP), 433

Shu, F. H., Najita, J. R., Shang, H., \& Li, Z.-Y. 2000, in Protostars and Planets IV, ed. V. Mannings, A. P. Boss, \& S. S. Russell (Tucson, AZ: Univ. Arizona Press), 789

Takahashi, S., Ho, P. T. P., Tang, Y.-W., Kawabe, R., \& Saito, M. 2009, ApJ, 704, 1459

Tang, Y.-W., Ho, P. T. P., Koch, P. M., \& Rao, R. 2010, ApJ, 717, 1262

Targon, C. G., Rodrigues, C. V., Cerqueira, A. H., \& Hickel, G. R. 2011, ApJ, 743,54

Tobin, J. J., Hartmann, L., Looney, L. W., \& Chiang, H.-F. 2010, ApJ, 712, 1010

Tomisaka, K. 2011, PASJ, 63, 147

Vaillancourt, J. E. 2006, PASP, 118, 1340 